\title{
Generalized analysis of a muffler with any number of interacting ducts
}

\author{
T. Kar, M.L. Munjal* \\ Facility for Research in Technical Acoustics, Department of Mechanical Engineering, Indian Institute of Science, \\ Bangalore-560 012, India
}

\begin{abstract}
The muffler elements that use perforated elements for acoustic attenuation are common in practice. In typical commercial mufflers perforated elements are used involving two, three, four or more interacting ducts. Analysis of such configurations involves writing down the basic governing equations of mass continuity, momentum balance, etc., and then elimination of velocity variables to obtain the coupled ordinary differential equations in terms of acoustic pressure variables. Mathematical modelling and the consequent analytical derivation of the transmission loss for these multi-duct acoustical elements become increasingly tedious, as just not the number of ducts, but also their relative arrangement along with the boundary conditions dictate the analysis considerably. In the present paper, authors have proposed a generalization and thus an algebraic algorithm to directly produce the system matrix, eliminating the tedium of writing the basic governing equations and elimination of velocity variables. Also, a convenient approach for applying the boundary conditions is outlined here.
\end{abstract}

\section{Introduction}

Analysis of perforated acoustic elements began with Sullivan and Crocker's [1] analytical prediction for a concentric-tube resonator. Later, Sullivan [2] proposed a segmentation method

\footnotetext{
*Corresponding author. Tel.: +918022932303; fax: + 918023600648.

E-mail address: munjal@mecheng.iisc.ernet.in (M.L. Munjal).
} 


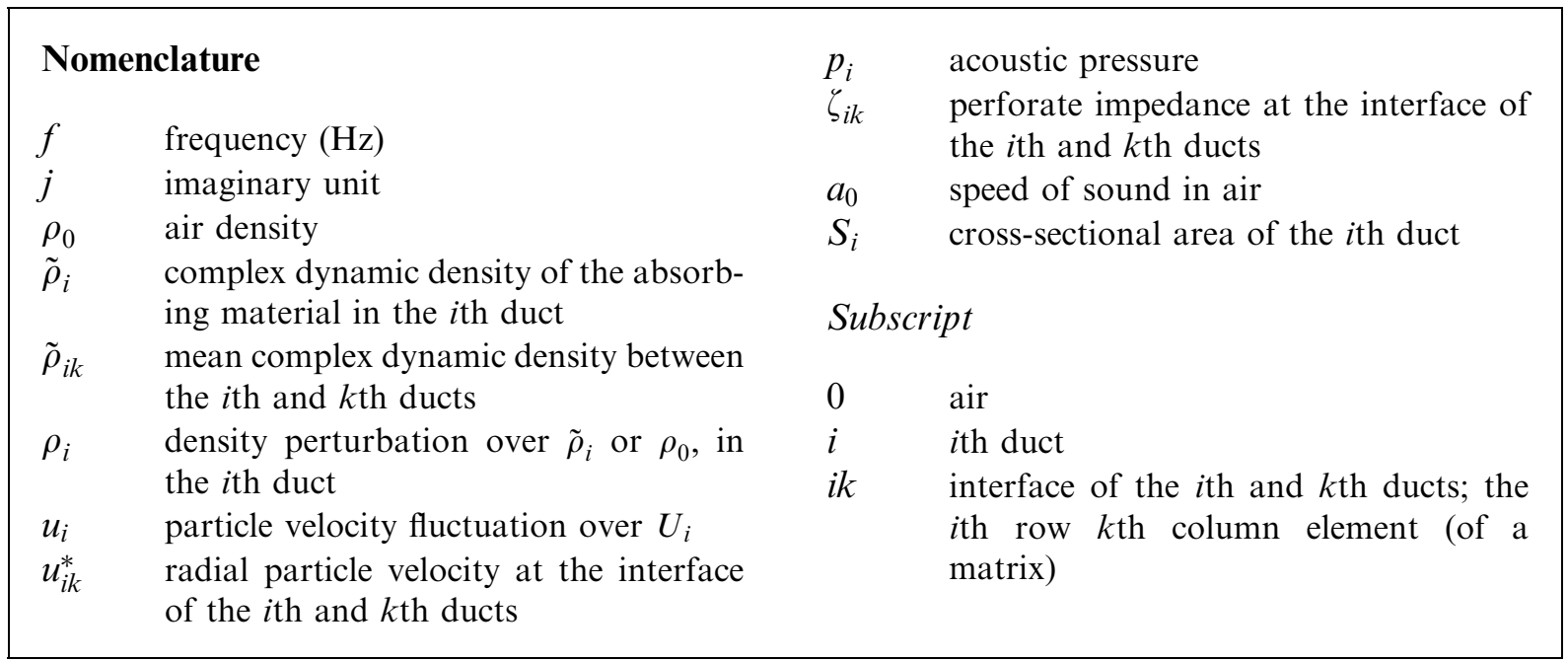

for the same configuration and extended it to three-duct elements, considering lumped admittance at discrete intervals along the perforate boundary, whereas successive branch points are separated by a rigid and uniform duct segment. Such lumped admittance elements separated by rigid tubular segments are described by unique transfer matrices and multiplied successively to arrive at the final form. On the negative side, it was the discretization of an essentially continuous process that kept it at bay.

Jayaraman and Yam [3] gave the first decoupling approach for a closed-form solution for a simple co-axial concentric-tube resonator. It has a limitation in the form of an assumed hypothetical situation of equal flow velocities in the two interacting ducts, which is obviously not a fact. Nevertheless, it may be perceived as the most effective method for any randomly configured acoustic element of parallel ducts with a stationary medium. Later Munjal et al. [4] produced a generalized decoupling method for analyzing the perforated elements. Application of different flow conditions in the distributed parameter method are described by Munjal [5] for both two- and three-duct elements as shown in Fig. 1.

Further attempts have been made to analyze similar acoustical elements for different configurations and flow conditions. In a recent development, Munjal [6] analyzed an axisymmetric pod silencer, making use of the bulk reaction model, where the pod segment may be treated as a three-duct element. Similarly, the wave interaction in four ducts are examined by Munjal [7] for the case of a flush-tube three-pass perforated element muffler.

The commonality among different tubular acoustical elements is the trend of governing differential equations and their affiliated coefficients. The number of coefficients increases with the number of interacting ducts, leading to involved algebraic calculations. It may be noticed that the number increases from eight for a simple concentric-tube configuration to fourteen for the three-duct perforated elements. To make things worse, coefficients vary with configurations of equal number of interacting tubes; i.e., the coefficients for an annular airgap lined duct are different from their counterparts of the reverse-flow or the cross-flow three-duct expansion chambers. 

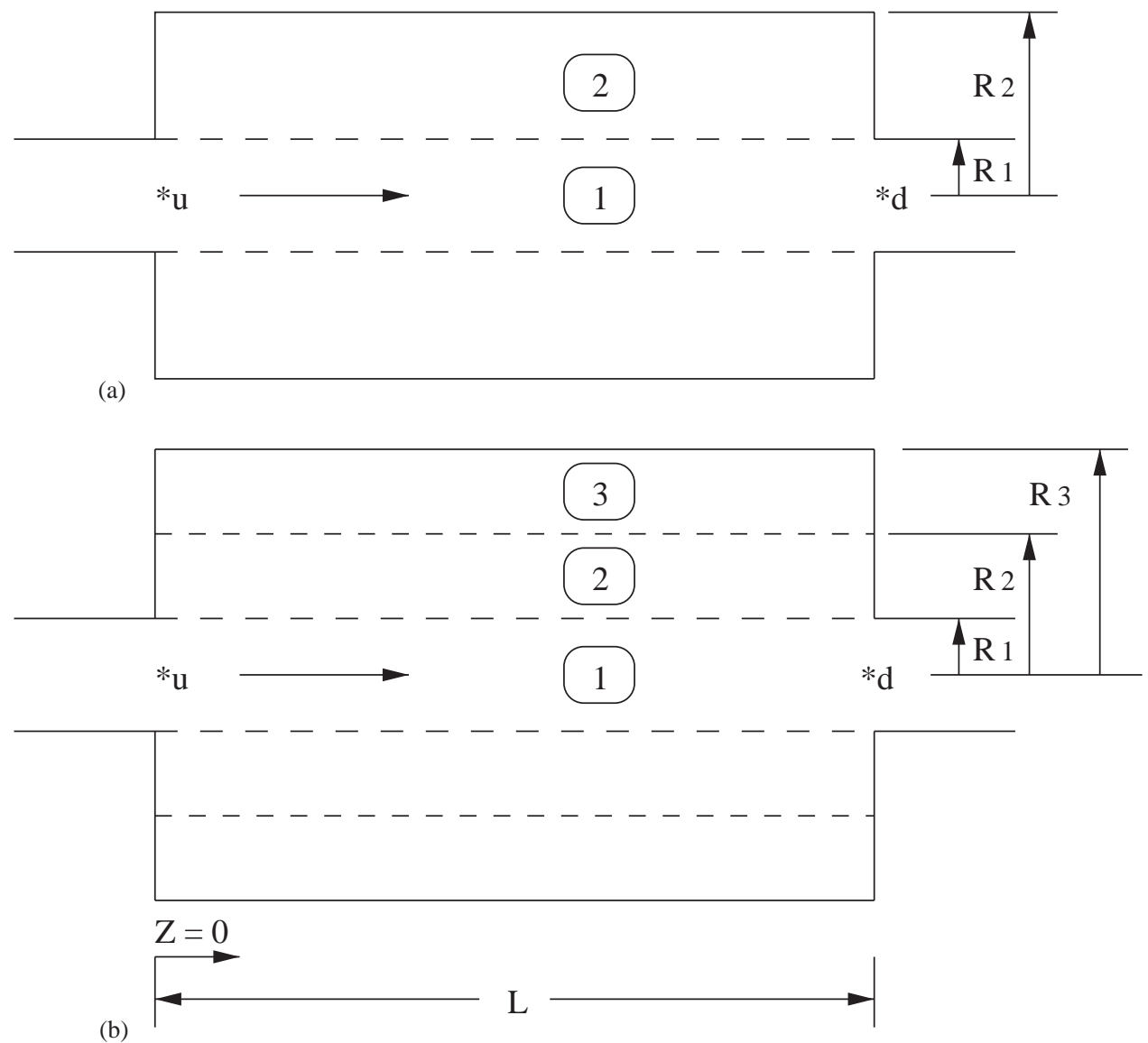

Fig. 1. (a) Two-duct and (b) three-duct concentric-tube resonators.

In the literature [8,9], the mass continuity equations associated with the interacting ducts for a dissipative silencer seem unreasonable, as they fail to satisfy the fundamental principle of the mass conservation between a pair of interacting finite control volumes. In this work, the continuity equation has been modified in order to meet the above criterion by incorporating a mean complex dynamic density $\tilde{\rho}_{i k}$, for the mass interaction between the $i$ th and $k$ th ducts. For a generality, it is rational to assume that, for all the ducts, the dynamic density $\tilde{\rho}_{i}$, wave number $k_{i}$ and characteristic impedance $Y_{i}$ are all complex numbers. This eventually accommodates the corresponding real values for a duct without any absorbing material and hence any nondissipative configuration.

It would therefore be desirable to develop an algebraic algorithm for modeling a configuration with " $n$ " interacting tubes or parallel ducts, which will provide a clarity of thought for completeness, and hence a generality, in a way, to preclude the tedious algebraic manipulations. 


\section{Methodology: a general algorithm}

The following analysis presumes linear plane waves in a uniform, inviscid incompressible mean flow. Specifically, the assumptions made in the formulation of the governing equations of motion for any perforated-tube muffler configuration are as follows:

1. Spatial variations of acoustic pressure and thence density, across the wave fronts in any duct are negligible.

2. Amplitudes of pressure and density perturbations in ducts are negligible compared to their mean values.

3. Grazing flow condition is applied to all ducts.

4. Temperature variation and viscous effect of the medium are negligible.

5. Perforation along the entire length of the tube is uniform.

6. Wall thicknesses of the ducts are negligible as compared to their radii.

7. The porous absorbent material of any $i$ th duct is considered as an equivalent fluid with complex wave number $k_{i}$ and characteristic impedance $Y_{i}$.

The associated sound propagation and attenuation is described next in terms of a set of randomly configured ' $n$ ' parallel tubes, with no limitation on their inter-connectivity. Then, the linearized equation for mass continuity of the $i$ th duct is given by

$$
\frac{D \rho_{i}}{D t}+\tilde{\rho}_{i} \frac{\partial u_{i}}{\partial z}+\sum \frac{P_{i k}}{S_{i}} u_{i k}^{*} \tilde{\rho}_{i k}=0
$$

and the corresponding generalized momentum equation for the same duct becomes

$$
\tilde{\rho}_{i} \frac{D u_{i}}{D t}+\frac{\partial p_{i}}{\partial z}=0 .
$$

Here, $\tilde{\rho}_{i}$ is the time-average component of the density for any tube and $\rho_{i}$ is the space average of the density perturbation over the corresponding cross-sectional area $S_{i}$. Likewise, $u_{i}$ is the velocity perturbation on $U_{i}$, the mean flow velocity in the $i$ th duct. Wave interaction between the $i$ th and $k$ th ducts is caused by $u_{i k}^{*}$, the radial particle velocity across their interface, $\tilde{\rho}_{i k}$, the average complex dynamic density, and $P_{i k}$ is the respective interface perimeter.

The fluctuating radial particle velocity through the perforated interface $u_{i k}^{*}$, as mentioned in Eq. (1), is a function of the pressure difference across the perforations, i.e., $p_{i}$ and $p_{k}$. Thus,

$$
u_{i k}^{*}=\frac{p_{i}(z)-p_{k}(z)}{\rho_{0} a_{0} \zeta_{i k}},
$$

where $\zeta_{i k}$ is the non-dimensional specific acoustic impedance of the perforates at the interface of the $i$ th and $k$ th ducts, and $a_{0}$ is the speed of sound in air. The mean complex dynamic density $\tilde{\rho}_{i k}$, at the interface of the $i$ th and $k$ th ducts may be given as

$$
\tilde{\rho}_{i k}=0.5\left(\tilde{\rho}_{i}+\tilde{\rho}_{k}\right) .
$$

Assuming an isentropic process in each of the ducts, the pressure and density perturbations in the $i$ th duct are related by

$$
p_{i}=\rho_{i} a_{i}^{2} .
$$


Assuming harmonic time dependence of all variables in Eqs. (1) and (2), and using Eqs. (3)-(5), yields a set of " $N$ " number of coupled differential equations. Hence, the corresponding wave equation for any $i$ th duct may be given as

$$
\begin{aligned}
& \left(1-M_{i}^{2}\right) p_{i}^{\prime \prime}-\left[2 \mathrm{j} k_{i} M_{i}+\sum U_{i} C_{i k}\right] p_{i}^{\prime}+\left[k_{i}^{2}-\sum \mathrm{j} \omega C_{i k}\right] p_{i} \\
& \quad+\left[\sum U_{i} C_{i k}\right] p_{k}^{\prime}+\left[\sum \mathrm{j} \omega C_{i k}\right] p_{k}=0,
\end{aligned}
$$

where the parametric constant $C_{i k}$ is given by

$$
C_{i k}=\frac{P_{i k} \tilde{\rho}_{i k}}{S_{i} \zeta_{i k} \rho_{0} a_{0}} .
$$

These wave equations may be arranged in a matrix as a set of simultaneous differential equation and may be given by

$$
[\mathbf{A}]\{p(z)\}=\{0\}
$$

where

$$
\{p(z)\}=\left[\begin{array}{llll}
p_{1}(z) & p_{2}(z) & \ldots & p_{n}(z)
\end{array}\right]^{\mathrm{T}}
$$

and $[\mathbf{A}]$ is an $N$ th order square matrix, given by

$$
[\mathbf{A}]=D^{2}\left[I_{n}\right]+D[\beta]+[\gamma]
$$

where $D$ is the differential operator $\mathrm{d} / \mathrm{d} z$. Then, the constituent elements of [A] may be given as

$$
[\mathbf{A}]_{i k}= \begin{cases}D^{2}+\beta_{i k} D+\gamma_{i k} & \text { if } i=k, \\ \beta_{i k} D+\gamma_{i k} & \text { if } i \neq k .\end{cases}
$$

Coefficients $\beta_{i k}$ and $\gamma_{i k}$ are constants, the expressions for which may be predicted heuristically as shown below.

Plane wave propagation in ducts (Eq. (10)) has the system matrix [A], with normalized coefficients $\gamma_{i k}$ and $\beta_{i k}$, which are parametric constants. Coefficients $\beta_{i k}$ 's provide the acoustic resistance to particle velocity, and are therefore analogous to damping in a vibrating system. These coefficients vanish for a stationary medium, leaving only $\gamma_{i k}$ 's. The total number of parametric coefficients (both $\beta_{i k}$ and $\gamma_{i k}$ ) increases with the number of interacting ducts and stands at ' $6 n-4$ ', whereas ' $n$ ' is the number of interacting tubular ducts. Irrespective of the limits and the boundary conditions (BCs), coefficients of the system matrix can be generalized and may be given as

$$
\begin{gathered}
\gamma_{i k}=\frac{\mathrm{j} \omega C_{i k}}{1-M_{i}^{2}}, \quad \beta_{i k}=-\frac{\mathrm{j} U_{i}}{k_{0}} \gamma_{i k} \quad \text { if } i \neq k, \\
\sum_{i=1}^{n} \gamma_{i k}=\frac{k_{i}^{2}}{1-M_{i}^{2}}, \quad \sum_{i=1}^{n} \beta_{i k}=\frac{-2 \mathrm{j} M_{i} k_{i}}{1-M_{i}^{2}} .
\end{gathered}
$$

Thus, the off-diagonal terms of the matrix [A] are known from Eq. (11), and then making use of Eq. (12), the diagonal terms may be calculated. Consequently, the coupled differential equations may be framed in a matrix form. 
Expressions (11) and (12) have been verified for all known two-duct, three-duct and four-duct configurations analyzed by Munjal et al. [4,5,7]. Further applications are given later in Section 3.

Reverting to the matrix equations (8), one can rearrange them as a set of ' $2 \times n=m$ ' simultaneous first-order equations. These may be written as

$$
\left[\begin{array}{cc}
{\left[-I_{n}\right]} & D\left[I_{n}\right] \\
D\left[I_{n}\right] & {[D \beta+\gamma]}
\end{array}\right]\{Y\}=\{0\}
$$

where $\left[I_{n}\right]$ is the $n$th order identity matrix, and

$$
\begin{gathered}
{[D \beta+\gamma]_{i k}=D \beta_{i k}+\gamma_{i k},} \\
\{Y\}_{i}= \begin{cases}\frac{\mathrm{d} p_{i}}{\mathrm{~d} z} & \text { for } i=1,2, \ldots, n, \\
p_{(i-n)} & \text { for } i=n+1, n+2, \ldots, m .\end{cases}
\end{gathered}
$$

Eq. (13) can be solved by making use of the standard eigen-matrix method [10]. Finally, the state vectors (pressures and particle velocities) at $z=0$ and $z=l$ may be rearranged and related by a forward transfer matrix [T], with elements $t_{i k}$ as follows:

$$
\{\Psi\}=[\mathbf{T}]\{\Omega\},
$$

where

$$
\{\Psi\}=\left[p_{1}, Y_{0} u_{1}, p_{2}, Y_{0} u_{2}, \ldots, p_{n}, Y_{0} u_{n},\right]_{z=0}^{\mathrm{T}},
$$

where $Y_{0}=\rho_{0} a_{0}$ is the characterstic impedance of the gaseous medium. Similarly, vector $\{\Omega\}$ can be framed corresponding to $z=l$.

In order to determine the transmission loss of the acoustical system, the transfer matrix [T] needs to be reduced systematically to a second-order square matrix using appropriate BCs $[5,7]$. Hence, given a system of ' $n$ ' interacting ducts, ' $m-2$ ' BCs distributed at the two ends may be made use of to derive the desired four pole parameters. BCs for such acoustical elements may be classified as follows:

1. State vectors (acoustic pressures and particle velocities) of two different ducts at the same end may be related in terms of a ' $2 \times 2$ ' transfer matrix. For example, state vectors for ducts 1 and 3 of Fig. 2, fused into the end chamber at $Z=L$, are related by the equivalent transfer matrix of the end chamber.

2. Acoustic admittance of a duct at the boundary.

Application of BCs of the first kind may use a methodical approach as shown by Munjal for a configuration with four interacting ducts [7]. Thus, all BCs of the first kind at one end can be applied before considering the ones at the other end. BCs of the second kind can always be given in terms of the admittance $\alpha$, at the axial boundaries of the ducts. These may be defined as

$$
\alpha=\frac{\rho_{0} a_{0} u_{i}}{p_{i}}
$$

for the $i$ th duct, and may have different values for the upstream and the downstream ends. 

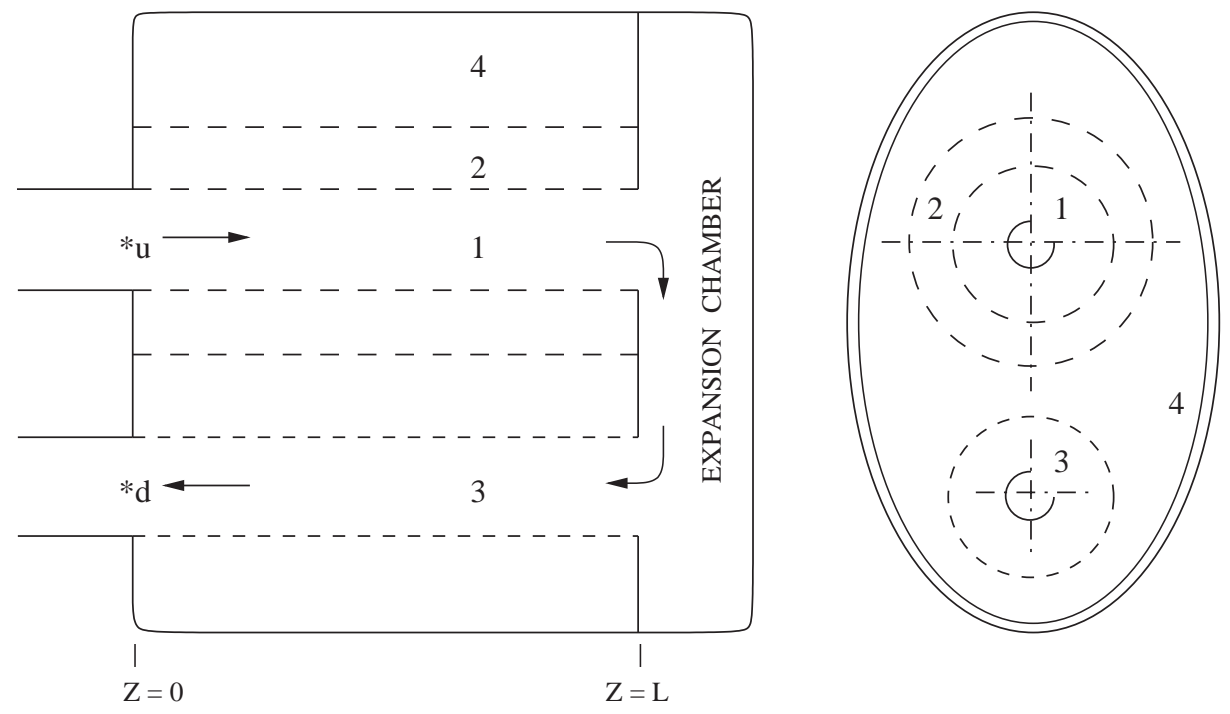

Fig. 2. Four-duct reverse flow muffler configuration.

In case of a rigid end, the particle velocity and hence the admittance becomes zero. Once all the $\mathrm{BCs}$ of the first kind are applied, a simple algorithm may be followed to apply the rest of the BCs as follows:

- In any muffler configuration with ' $n$ ' interacting ducts, there will be a few ducts with BCs of the second type applicable at both the ends. Pressure and velocity components of one such duct with known admittances are pushed down to occupy the $(m-1)$ th and $m$ th positions, respectively, for both the downstream vector, $\{\Psi\}$ and upstream vector, $\{\Omega\}$. Thus, applying those $\mathrm{BCs}$, the number of simultaneous equations will reduce to ' $m-2$ ' and they may be rewritten as

$$
\Psi_{k}=\sum_{i=1}^{m-2}\left[t_{k i}+\delta_{k}\left\{\alpha_{u} t_{(m-1) i}-t_{m i}\right\}\right] \Omega_{i}, \quad 1 \leqslant k \leqslant m-2,
$$

where $\delta_{k}$ is given as

$$
\delta_{k}=\frac{t_{k(m-1)}+\alpha_{d} t_{k m}}{\left[\left\{t_{m(m-1)}+\alpha_{d} t_{m m}\right\}-\alpha_{u}\left\{t_{(m-1)(m-1)}+\alpha_{d} t_{(m-1) m}\right\}\right]},
$$

where $\alpha_{u}$ and $\alpha_{d}$ are the upstream admittance and the downstream admittance, respectively.

- Repeating the process successively for all such ducts will leave the truncated transfer matrix operating over a vector of four variables. During this cyclic process one has to ensure that the value of the index ' $m$ ' is reduced by two after every cycle and again the elements of the transfer matrix $[\mathbf{T}]$ be replaced by the corresponding ones from Eq. (19). 
If, at the final step, the BCs do not relate to the same duct (as for the case of cross flow), the process of reduction may still be applied in a similar fashion, ensuring that the set of state variables relevent to those BCs are placed at the bottom half of both the vectors.

\subsection{Acoustic impedance of the perforates}

In some applications, fiber-based porous sound-absorbing materials are used in either duct across an interface. One such case arises when different backing materials are used in both the annular cavities in a three-duct concentric-tube resonator of Fig. 1. The perforate impedance for such configuration with a default stationary medium in either of the ducts may be modified and extended from the expression of the acoustic impedance of perforations facing backing material at one side given by Kirby and Cumming [11] and Selamet [9]. The impedance $\zeta_{i k}=\mathfrak{R}+\mathrm{j} \mathfrak{I}$ may be given as

$$
\zeta_{i k}=\left[6 \times 10^{-3}+\mathrm{j} k_{0}\left(t_{h}+0.375\left(\frac{k_{i} Y_{i}}{k_{0} Y_{0}}+\frac{k_{k} Y_{k}}{k_{0} Y_{0}}\right) d_{h}\right)\right] / \sigma,
$$

where $k_{i}$ and $Y_{i}$ are complex wave number and characteristic impedance of the acoustic lining with indices $i$ and $k$ representing the ducts across the interface. The corresponding grazing flow perforate impedance is given by [12]

$$
\zeta_{i k}=\left[0.00734\left(1+72.23 M_{i}\right)+\mathrm{j} 2.27 \times 10^{-3}\left(1+51 t_{h}\right)\left(1+\frac{k_{k} Y_{k}}{k_{0} Y_{0}}\right) d_{h} f\right] / \sigma .
$$

If the medium in the $k$ th duct is a sound-absorbing material, then $Y_{k}=Y_{w}$ and $k_{k}=k_{w}$. For any such sound-absorbing material, $Y_{w}$ and $k_{w}$ are precisely outlined by the empirical formulae of Delany and Bazley [13], later improved through modifications by Mechel [14]:

$$
\begin{gathered}
\frac{Y_{w}}{Y_{0}}= \begin{cases}1+0.0485(A)^{0.754}-\mathrm{j} 0.087(A)^{0.73}, & \text { if } A \leqslant 60, \\
\frac{0.5(A / \pi)+\mathrm{j} 1.4}{-1.466+\mathrm{j} 0.212 A^{0.5},} & \text { if } A>60,\end{cases} \\
\frac{k_{w}}{k_{0}}= \begin{cases}1+0.0978(A)^{0.6929}-\mathrm{j} 0.189(A)^{0.6185}, & \text { if } A \leqslant 60, \\
-1.466+\mathrm{j} 0.212 A^{0.5}, & \text { if } A>60 .\end{cases}
\end{gathered}
$$

Here $A$, the normalized flow resistivity of a $\lambda$-deep acoustic lining, is given by $A=E \lambda / Y_{0}$, whereas $E$ is the flow resistivity of the lining material.

Expressions for complex characteristic impedance and wavenumber in Eqs. (23) and (24) are such as to reduce to $Y_{0}$ and $k_{0}$, respectively, for zero flow resistivity (absence of the absorptive material). Still more general and precise formulae may be found in Ref. [15]. 


\section{Applications}

In order to demonstrate generality of the generalized algorithm, a few hypothetical muffler configurations shown in Figs. 2-4 are analyzed here. The generalized coefficient matrices are obtained for each of the configurations in accordance with the algorithm enunciated above. For each of the cases given below, the basic task is to construct the coefficient matrix [ $\gamma]$ of Eq. (9). With the matrix $[\gamma]$ thus constructed for any given perforated-tube acoustic element, diagonal terms $\gamma_{i i}$ may be derived by making use of Eq. (12) and so also the matrix [ $\left.\beta\right]$. Once the matrices $[\gamma]$ and $[\beta]$ are generated, solution to the system will follow as described above (Eqs. (13)-(20)).

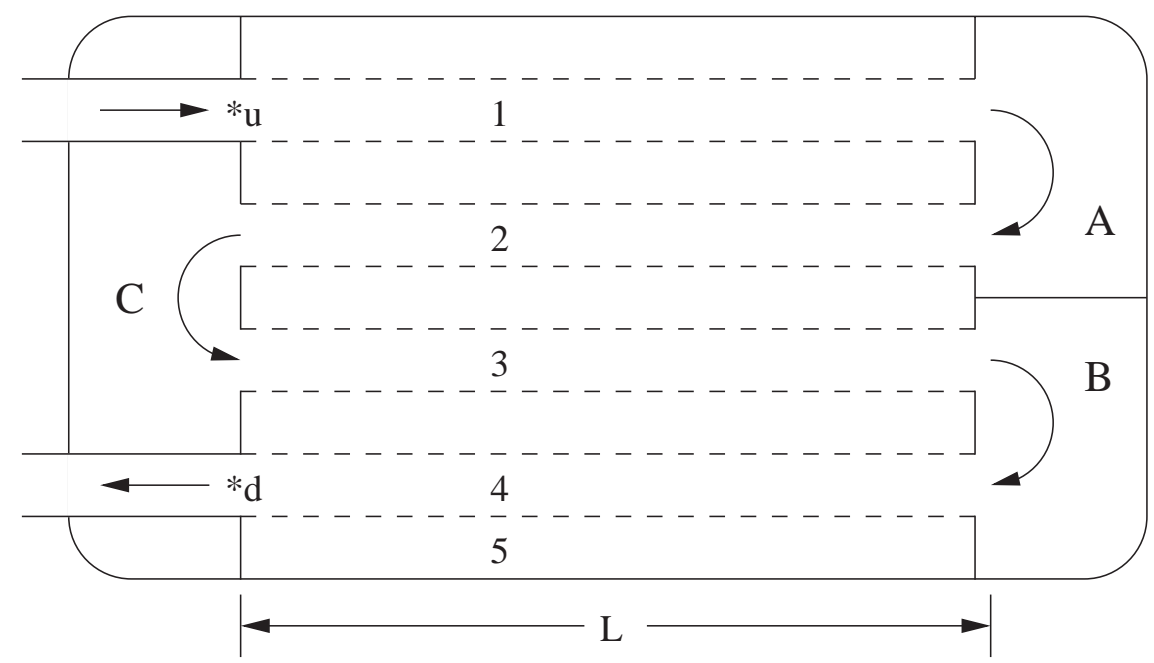

(a)

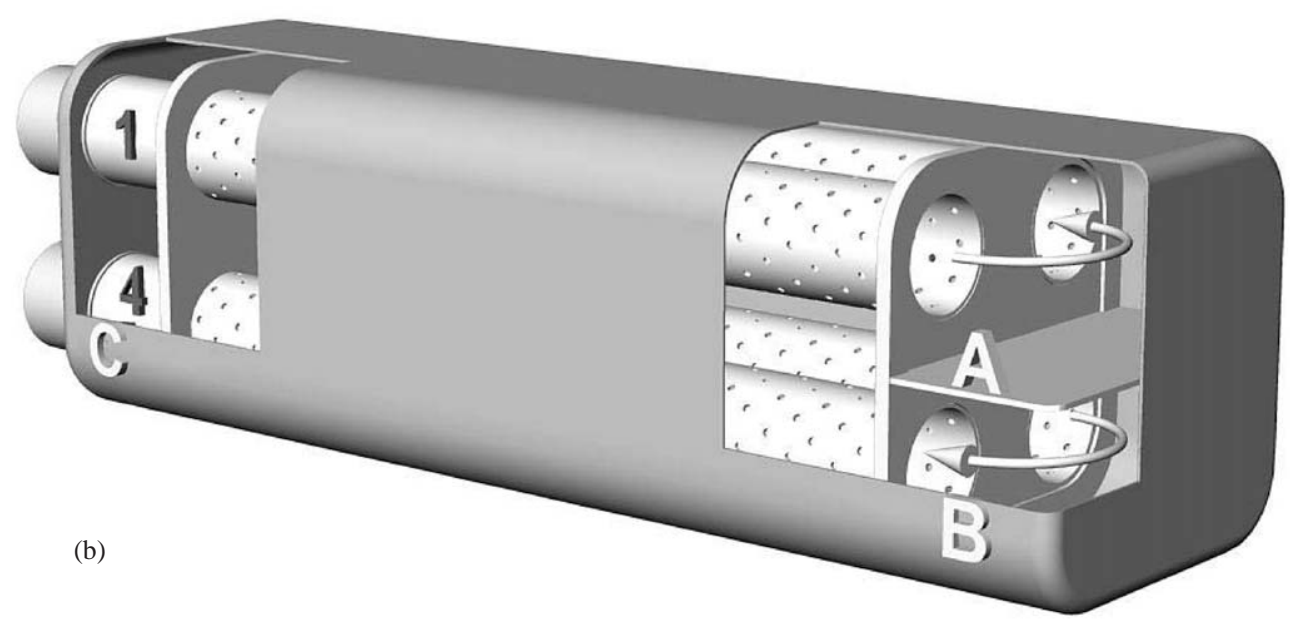

Fig. 3. Five-duct reverse flow concentric-tube resonator. (a) Schematic diagram, (b) the corresponding 3-D solid model. 


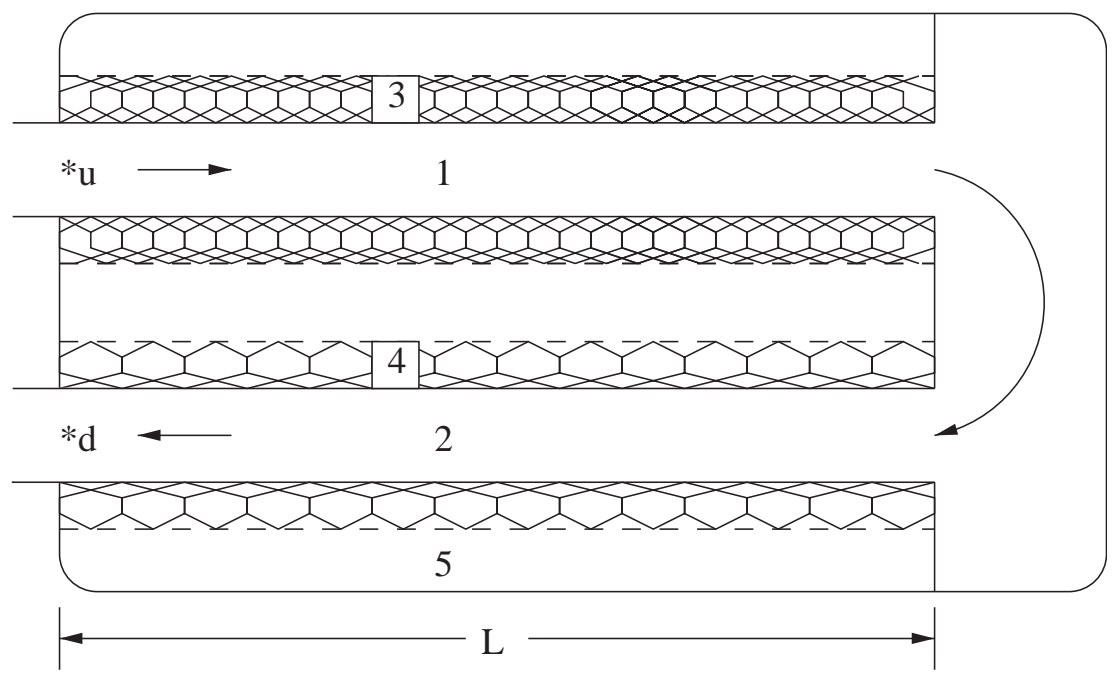

Fig. 4. Five-duct reverse flow concentric-tube resonator.

\subsection{Four-duct reverse flow}

Fig. 2 is basically a reverse-flow four-duct configuration that comprises all but one perforated tube, arranged in an elliptical shell (duct-4), fused into an expansion chamber at one end. A grazing flow exists in the ducts enumerated 1 and 3 in Fig. 2, whereas the media in the shell and the annular cavity (duct-2) are assumed stationary.

The generalized algorithm is put to use as in Eqs. (11) and (12) to articulate the coefficient matrix $[\gamma]$ of Eq. (9), and it may be given as

$$
[\gamma]=\left[\begin{array}{cccc}
\gamma_{11} & \frac{\mathrm{j} 8 \pi f}{a_{0} d_{1} \zeta_{12}\left(1-M_{1}^{2}\right)} & 0 & 0 \\
\frac{\mathrm{j} 8 \pi f d_{1}}{a_{0}\left(d_{2}^{2}-d_{1}^{2}\right) \zeta_{12}} & \gamma_{22} & 0 & \frac{\mathrm{j} 8 \pi f d_{2}}{a_{0}\left(d_{2}^{2}-d_{1}^{2}\right) \zeta_{24}} \\
0 & 0 & \gamma_{33} & \frac{\mathrm{j} 8 \pi f}{a_{0} d_{3} \zeta_{34}\left(1-M_{3}^{2}\right)} \\
0 & \frac{\mathrm{j} 2 \pi^{2} f d_{2}}{a_{0} S_{4} \zeta_{24}} & \frac{\mathrm{j} 2 \pi^{2} f d_{3}}{a_{0} S_{4} \zeta_{34}} & \gamma_{44}
\end{array}\right] .
$$

The diagonal element $\gamma_{i i}$ may be evaluated readily by means of Eq. (12).

\subsection{Five-duct reverse flow (flush-tube four-pass perforated element chamber)}

As shown in Fig. 3, a five-duct reverse flow perforated tube resonator consists of a set of four perforated tubes, and an enclosing shell. The flow is set in motion with duct 1, that expands into the expansion chamber (cavity) "A". As the flow grazes through the rest of the perforated tubes 
and the end cavities as shown in the virtual 3-D solid model, sound attenuates due to the combined effect of the impedances offered by the perforated tubes and the sudden area changes. The generalized coefficient matrix $[\gamma]$ for the common perforated portion may be given as

$$
[\gamma]=\left[\begin{array}{ccccc}
\gamma_{11} & 0 & 0 & 0 & \frac{\mathrm{j} 8 \pi f}{a_{0} d_{1} \zeta_{15}\left(1-M_{1}^{2}\right)} \\
0 & \gamma_{22} & 0 & 0 & \frac{\mathrm{j} 8 \pi f}{a_{0} d_{2} \zeta_{25}\left(1-M_{2}^{2}\right)} \\
0 & 0 & \gamma_{33} & 0 & \frac{\mathrm{j} 8 \pi f}{a_{0} d_{3} \zeta_{35}\left(1-M_{3}^{2}\right)} \\
0 & 0 & 0 & \gamma_{44} & \frac{\mathrm{j} 8 \pi f}{a_{0} d_{4} \zeta_{45}\left(1-M_{4}^{2}\right)} \\
\frac{\mathrm{j} 2 \pi^{2} d_{1} f}{a_{0} S_{5} \zeta_{15}} & \frac{\mathrm{j} 2 \pi^{2} d_{2} f}{a_{0} S_{5} \zeta_{25}} & \frac{\mathrm{j} 2 \pi^{2} d_{3} f}{a_{0} S_{5} \zeta_{35}} & \frac{\mathrm{j} 2 \pi^{2} d_{4} f}{a_{0} S_{5} \zeta_{45}} & \gamma_{55}
\end{array}\right] .
$$

As indicated earlier, $\gamma_{i i}$ may be evaluated readily by means of Eq. (12).

\subsection{Five-duct reverse flow (flush-tube two-pass perforated element chamber) with absorptive layers}

The configuration in Fig. 4 differs from the four-pass element in as much as fiber-based porous sound-absorbing materials are used in the concentric cavities surrounding the perforated tubes for a better performance in a selected frequency range. Use of the generalized algorithm yields the following coefficient matrix $[\gamma]$ :

$$
[\gamma]=\left[\begin{array}{ccccc}
\gamma_{11} & 0 & \frac{\mathrm{j} 8 \pi f \tilde{\rho}_{13}}{Y_{0} d_{1} \zeta_{13}\left(1-M_{1}^{2}\right)} & 0 & 0 \\
0 & \gamma_{22} & 0 & \frac{\mathrm{j} 8 \pi f \tilde{\rho}_{24}}{Y_{0} d_{2} \zeta_{24}\left(1-M_{2}^{2}\right)} & 0 \\
\frac{\mathrm{j} 2 \pi^{2} f d_{1} \tilde{\rho}_{13}}{Y_{0} S_{3} \zeta_{13}} & 0 & \gamma_{33} & 0 & \frac{\mathrm{j} 2 \pi^{2} f d_{3} \tilde{\rho}_{35}}{Y_{0} S_{3} \zeta_{35}} \\
0 & \frac{\mathrm{j} 2 \pi^{2} f d_{2} \tilde{\rho}_{24}}{Y_{0} S_{4} \zeta_{24}} & 0 & \gamma_{44} & \frac{\mathrm{j} 2 \pi^{2} f d_{4} \tilde{\rho}_{45}}{Y_{0} S_{4} \zeta_{45}} \\
0 & 0 & \frac{\mathrm{j} 2 \pi^{2} f d_{3} \tilde{\rho}_{35}}{Y_{0} S_{5} \zeta_{35}} & \frac{\mathrm{j} 2 \pi^{2} f d_{4} \tilde{\rho}_{45}}{Y_{0} S_{5} \zeta_{45}} & \gamma_{55}
\end{array}\right] .
$$

Again, $\gamma_{i i}$ may be evaluated by means of Eq. (12). Here it may be noted that $\tilde{\rho}_{13}=\tilde{\rho}_{35}$ and $\tilde{\rho}_{24}=\tilde{\rho}_{45}$ for the above configuration (Fig. 4).

\section{Concluding remarks}

The generalized algebraic algorithm presented here for perforated element mufflers results in great convenience and minimizes oversights or human errors that often accompany complex 
mathematical modeling. Most importantly, the algorithm for applying the BCs holds good for all acoustical configurations. The generalized algorithm presented in this paper applies to dissipative ducts as well as those filled up with the gaseous medium (usually air). However the physical model of Refs. [1-5] implied in the algorithm may not hold exactly for dissipative ducts as shown in Ref. [16], although it has been applied with some success in Refs. [8,9].

\section{Acknowledgements}

The authors would like to place on record their appreciation of the financial sponsorship of FRITA by the Department of Science and Technology of the Government of India.

\section{References}

[1] J.W. Sullivan, M.J. Crocker, Analysis of concentric tube resonators having unpartitioned cavities, Journal of the Acoustical Society of America 64 (1) (1978) 207-215.

[2] J.W. Sullivan, A method for modeling perforated tube muffler components-Part I: theory, Journal of the Acoustical Society of America 66 (3) (1979) 772-778.

[3] K. Jayaraman, K. Yam, Decoupling approach to modeling perforated tube muffler components, Journal of the Acoustical Society of America 69 (2) (1981) 390-396.

[4] M.L. Munjal, K.N. Rao, A.D. Sahasrabudhe, Aeroacoustic analysis of perforated muffler components, Journal of Sound and Vibration 114 (2) (1987) 173-188.

[5] M.L. Munjal, Acoustics of Ducts and Mufflers, Wiley, New York, 1987.

[6] M.L. Munjal, Analysis and design of pod silencers, Journal of Sound and Vibration 262 (2003) $497-507$.

[7] M.L. Munjal, Analysis of a flush-tube three-pass perforated muffler by means of transfer matrices, International Journal of Sound and Vibration 2 (2) (1997) 63-68.

[8] Chao-Nan Wang, Numerical decoupling analysis of a resonator with absorbent material, Applied Acoustics 58 (1999) 109-122.

[9] A. Selamet, I.J. Lee, Z.L. Ji, N.T. Huff, Acoustic attenuation performance of perforated absorbing silencers, $S A E$ Noise and Vibration Conference, Paper 2001-01-1435, 2001.

[10] K.S. Peat, A numerical decoupling analysis of perforated pipe silencer elements, Journal of Sound and Vibration 128 (2) (1988) 199-212.

[11] R. Kirby, A. Cummings, The impedance of perforated plate subjected to grazing gas flow and backed by porous media, Journal of Sound and Vibration 217 (4) (1998) 619-636.

[12] K.N. Rao, M.L. Munjal, Experimental evaluation of impedance of perforates with grazing flow, Journal of Sound and Vibration 108 (2) (1986) 283-295.

[13] M.E. Delany, B.N. Bazley, Acoustical characteristics of fibrous absorbent material, Applied Acoustics 3 (1970) 106-116.

[14] F.P. Mechel, Extension of low frequencies of the formulae of Delany and Bazley for absorbing materials, Acustica 35 (1976) 210-213 (in German).

[15] F.P. Mechel, Formulas of Acoustics, Springer, Berlin, 2002.

[16] S.N. Panigrahi, M.L. Munjal, Comparison of various methods for analyzing lined circular ducts, Journal of Sound and Vibration, in press; doi:10.1016/j.jsv.2004.09.006. 